\title{
Letter To The Editor: Amylase Isoenzymes in Cystic Fibrosis
}

\author{
GORDON G. FORSTNER AND GEOFFREY DAVIDSON
}

The Hospital for Sick Children, Toronto, Ontario, Canada and The Adelaide Children's Hospital, North Adelaide, S. Australia

\section{REPLY TO DRS. R. O. WOLF AND L. TAUSSIG}

No one wishes to misquote someone else. We have, therefore, taken the very serious accusations of Drs. Wolf and Taussig to heart and carefully reassessed our assessment of their paper in $\mathbf{J}$. Lab. Clin. Med., 87: 164, (1976).

We stated in our paper that Wolf et al. (2) had found that cystic fibrosis (CF) patients with pancreatic insufficiency had a rather low total serum amylase activity. Perhaps we should have quoted verbatim from their summary "The mean total serum amylase of the CFP patients with PI was significantly lower than the control group." We wish to make no judgment as to whether all of the values for the CFP group were within the normal range, and, indeed, can't see that it makes much difference because the argument, if one exists, has to do with a statistical comparison of the means. The purpose of our paper was to present evidence, based on the determination of total serum amylase levels in four groups of patients, and qualitative isoelectric focusing patterns in representative individuals, that suggests that patients with $\mathrm{CF}$ have an absolute increase, not a percent increase, in circulating salivary isoamylase. We cannot agree that we have misinterpreted the data of Wolf et al. (2) or that we have confirmed their work. As indicated in their letter, they found that the percent contribution of salivary amylase was higher in their patients which CF and pancreatic insufficiency, which is not surprising considering the reduced pancreatic isoamylase, whereas our contention is that total salivary isoamylase activity is higher in these patients.

The crucial question is whether Wolf and Taussig are right in their view that "the changes in serum amylase values in patients with CF are on the average due to changes in the pancreatic contribution to the serum." Such must be the case if they are right in their quantitative assessment that variations in the serum salivary isoamylase values (absolute values) are not significantly different from the normal. As we took care to point out in our paper, there is no uniformity of opinion in the literature on this subject and in fact we are not alone in providing data that indicate that the salivary isoamylase is elevated. A great deal depends on the validity of the assay methods in use. We did not attempt quantitation, and would be unwilling to draw conclusions from the small variations in the isoenzyme patterns shown in our figures, especially on the basis of the few patients we have examined. In attempting to come to grips with the data of Wolf $e t$ al. (2), we simply suggested that some of the difference may have been due to feeding because we had noted slightly higher (albeit not always significant) total mean values in our postprandial controls and in our Shwachman's patients. It is still true, as far as we are concerned, that in the study of Wolf et al. (2) "the total recovery for each gel was $50 \%$ of the total amylase activity applied." At least, that is what is written in the description of the method in their paper. In order to correct for this loss over the period of $48 \mathrm{hr}$ required for the assay of enzyme activity Wolf $e t$ al. (2) state that they assumed that the rate of decay for pancreatic and salivary isoamylase was the same. The justification for doing so was that they observed similar isoenzyme ratios to those reported by other groups using different methods. This may be true, but it is always difficult to accept quantitative data when $50 \%$ of the total enzyme activity is lost during the assay.

We are indeed sorry that we substituted the figure of $30 \%$ for $39 \%$ as the mean pancreatic isoamylase activity estimated by Wolf et al. (2) in the CF patients with steatorrhea. The error was certainly not self-serving. The larger percent provides even more support for our suggestion that these levels of pancreatic isoamylase are much higher than one would expect from our isoelectric focusing patterns or the experience of others (1).

Finally, we agree that our case would benefit from direct confirmation by quantitative estimation of isoenzymes. We so stated in our paper. We are currently attempting to develop column isoelectrofocusing techniques that are both quantitative and adaptable to the assay of satisfactorily large numbers of samples.

\section{REFERENCES AND NOTES}

1. Skude, G.: Sources of the serum isoamylases and their normal range of variation with age. Scand. J. Gastroenterol., 10: 577 (1975)

2. Wolf, R. O., Taussig, L. M., Ross, M. E., and Wood, R. E.: Quantitative evaluation of serum pancreatic isoamylases in cystic fibrosis. J. Lab. Clin. Med., 87: 164 (1976)

3. Received for publication April 19, 1979.

4. Accepted for publication April 20, 1979 